\title{
Study on novel structure of Mn complex, $\mathrm{C}_{24} \mathrm{H}_{18} \mathrm{CrMnN}_{4} \mathrm{O}_{5}$
}

\author{
Ying Liu ${ }^{1}$, Haixing Liu $^{1 *}$ \\ ${ }^{1}$ College of Chemistry and Chemical and Environmental Engineering, Weifang University, Weifang 261061, P.R. China
}

\begin{abstract}
The novel Mn complex $\mathrm{C}_{24} \mathrm{H}_{18} \mathrm{CrMnN}_{4} \mathrm{O}_{5}$ was investigated by hydrothermal and its crystal structure was characterized using X-ray diffraction technology. The $\mathrm{Mn}$ atom is six coordinated by four $\mathrm{N}$ atom from two 1,10-phenanthroline and two $\mathrm{O}$ atoms from $\mathrm{CrO}_{4}^{-}$. The hydrogen bonding $\mathrm{O}-\mathrm{H}$...O had central effect for crystal stability.
\end{abstract}

\section{Introduction}

The diversity of metal-organic coordination structures will have certain advantages in photochemistry, catalysis, absorptivity, biological properties, magnetism and electrical properties [1-3]. Because manganese (II) complexes often exist in the center of the activity of enzymes (superoxide dismutase, peroxidase, aminopeptidase, sialyl and fucosyl transferase), the investigation of manganese (II) complexes is a very meaningful work [4-7]. The Mn (II) biological activity may be related to the interaction of carboxylate ligands and manganese. The carboxylate ligands are easy to form binuclear, trinuclear and high nuclear compounds [8]. 1,10 -phenanthroline is major chelating agent for the formation of various supramolecular structures. Yang Hongli et al [9] synthesized two silver and cadmium compounds of $\operatorname{Ag}(1,2,4$-benzenetricarboxylic acid)(2,2'-bipyridine) and $\mathrm{Cd}(1,2,4$-benzenetricarboxylic acid) $(2,2$ '-bipyridine $\left.)\left(\mathrm{H}_{2} \mathrm{O}\right)_{2}\right]_{\mathrm{n}}$ by hydrothermal and studied their structure and property by X-ray single crystal diffraction, thermal gravimetric analysis, IR spectra and fluorescence spectroscopy. The three Co metal-organic frameworks compounds ([Co(2,6-di(1H-imidazol-1-yl)naphthalene)(5-aminoisop hthalic acid) $] \cdot 2 \mathrm{DMF}$

[Co(2,6-di(1H-imidazol-1-yl)naphthalene)(5-aminoisopht halic acid)]·DMF, [Co(2,6-di(1H-imidazol-1-yl)naphthalene)(4,4'-iminodib enzoic

acid $\left.)\left(\mathrm{H}_{2} \mathrm{O}\right)_{2}\right] \cdot 0.5(2,6$-di(1H-imidazol-1-yl)naphthalene). $\mathrm{H}_{2} \mathrm{O}$ ) were synthesized by Liu et al [10] using solvothermal method. The compounds were analysized again with powder X-ray diffraction analyses, infrared spectra, single-crystal and elemental analyses. Zhang Li et al [11] explored new cobalt compound [Co(pyridine-2,6-dicarboxylic
acid)(2,2'-bipyridine)Cl] $\mathrm{C}_{2} \mathrm{H}_{5} \mathrm{OH}$ using hydrothermal condition and characterized by $\mathrm{X}$-ray single crystal diffraction and infrared spectroscopy. The material charge distribution, electrostatic potential and relevant electronic properties were analyzed by density functional theory calculations method. In this paper, the novel $\mathrm{Mn}$ complex is reported.

\section{Experimental}

The mixture of $\mathrm{MnSO}_{4} \quad(0.1 \mathrm{mmol}, 0.015 \mathrm{~g})$, 1,10-phenanthroline $(0.1 \mathrm{mmol}, 0.02 \mathrm{~g}), \mathrm{K}_{2} \mathrm{CrO}_{4}(0.1 \mathrm{mmol}$, $0.02 \mathrm{~g})$ and $\mathrm{Na}_{2} \mathrm{~B}_{4} \mathrm{O}_{7} 10 \mathrm{H}_{2} \mathrm{O}(0.1 \mathrm{mmol}, 0.04 \mathrm{~g})$ were added into $10 \mathrm{~mL}$ water (containing methanol(4/10, v/v)) with heated for $8 \mathrm{~h}$ at $414 \mathrm{~K}$. The solution was obtained by filtration after cooling the reaction to room temperature. Black block single crystals was got for X-ray analysis after weeks.

\section{Results and discussions}

Fig.1 is novel compound crystal sketch map. It composed of Mn cation, 1,10-phenanthroline, chromate anion and water molecular. The Table 1 lists the crystal compound refinement data. The $\mathrm{Mn}$ atom is six coordinated by four $\mathrm{N}$ atom from two 1,10-phenanthroline and two $\mathrm{O}$ atoms from $\mathrm{CrO}_{4}^{-}$. The bond lengths $\mathrm{d}(\mathrm{Mn}-\mathrm{N})$ are in the range of 2.266-2.355 $\AA$. The $\mathrm{d}(\mathrm{Mn}-\mathrm{O})$ are 2.067 and $2.108 \AA$. The bond angles of O1-Mn1-O2\#, O1-Mn1-N2, N1-Mn1-N3 are 90.1, 94.5, 88.7 , respectively. The torsion angles of O2\#-Mn1-O1-Cr1, N3-Mn1-O1-Cr1, O4-Cr1-O1-Mn1 are 38.4, 129.5, 78.3 ${ }^{\circ}$, respectively. The Table 2 lists the section bond lengths and angles.

The crystal packing is stabilized by hydrogen bond interaction. 


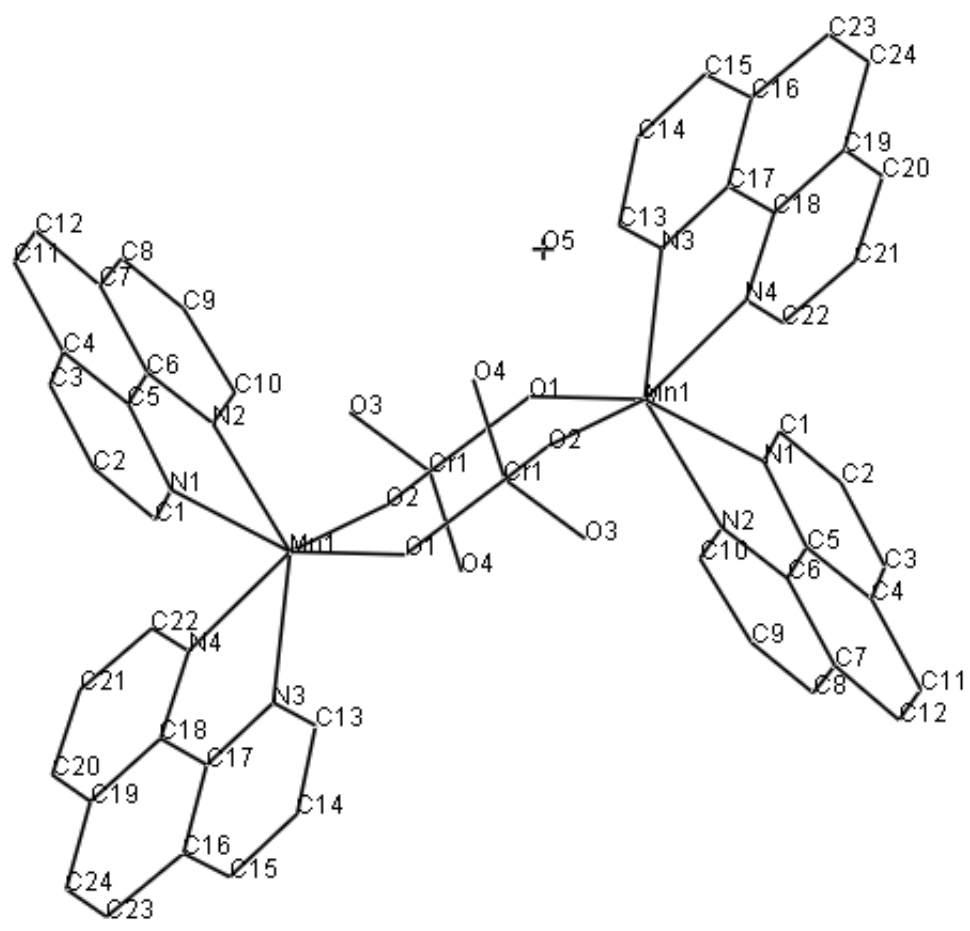

Fig.1 The crystal structure of $\mathrm{C}_{24} \mathrm{H}_{18} \mathrm{CrMnN}_{4} \mathrm{O}_{5}$

Table 1. Crystal compound refinement data

\begin{tabular}{ll} 
Empirical formula & $\mathrm{C}_{24} \mathrm{H}_{18} \mathrm{CrMnN} \mathrm{O}_{5}$ \\
Weight & 549.36 \\
$\mathrm{~T}$ & $293(2) \mathrm{K}$ \\
$\lambda$ & $0.71073 \AA$ \\
System, group & Monoclinic, $\mathrm{P} 2(1) / \mathrm{c}$ \\
Cell & $\mathrm{a}=9.3421(19) \AA \quad$ alpha $=90^{\circ}$. \\
& $\mathrm{b}=13.021(3) \AA \quad$ beta $=93.388(3)^{\circ}$. \\
& $\mathrm{c}=18.543(4) \AA \quad$ gamma $=90^{\circ}$. \\
Volume & $2251.7(8) \AA^{3}$ \\
Z, density & $4,1.621 \mathrm{Mg} / \mathrm{m}^{3}$ \\
Absorpting number & $1.089 \mathrm{~mm}^{-1}$ \\
$\mathrm{~F}$ & 1116 \\
Size & $0.38 \mathrm{x} 0.35 \mathrm{x} 0.32 \mathrm{~mm}$ \\
Collecting range & 3.01 to $25.01^{\circ}$. \\
Limiting indices & $-10<=\mathrm{h}<=11,-15<=\mathrm{k}<=15,-22<=1<=22$ \\
Reflection collected (unique) & $16157 / 3935[\mathrm{R}($ int $)=0.1766]$ \\
Completeness to theta(25.02) & $99.2 \%$ \\
Correcting correction & Semi-empirical from equivalents \\
Transmissing & 0.7220 and 0.6824 \\
Refining & Full-matrix least-squares on $\mathrm{F}^{2}$ \\
Data / restraints / parameters & $3935 / 0 / 317$ \\
GoF & 1.285 \\
Final R indices $[\mathrm{I}>2$ sigma(I) $]$ & $\mathrm{R} 1=0.1624, \mathrm{wR} 2=0.3920$ \\
R indices (all data) & $\mathrm{R} 1=0.2515, \mathrm{wR} 2=0.4412$ \\
Extinction coefficient & $0.007(4)$ \\
Largest diff. peak and hole & 2.849 and $-1.016 \mathrm{e} . \AA^{-3}$ \\
\hline &
\end{tabular}

Table 2. The section bond lengths and angles ([ $\left.\left.\AA],{ }^{\circ}\right]\right)$

$\begin{array}{cc}\operatorname{Mn}(1)-\mathrm{O}(1) & 2.067(10) \\ \mathrm{Mn}(1)-\mathrm{N}(2) & 2.266(13) \\ \mathrm{Mn}(1)-\mathrm{N}(3) & 2.299(13) \\ \mathrm{Mn}(1)-\mathrm{N}(1) & 2.344(12) \\ \mathrm{Mn}(1)-\mathrm{N}(4) & 2.355(14) \\ \mathrm{Cr}(1)-\mathrm{O}(3) & 1.580(10)\end{array}$




$\begin{array}{cc}\mathrm{Cr}(1)-\mathrm{O}(4) & 1.636(12) \\ \mathrm{Cr}(1)-\mathrm{O}(2) & 1.651(11) \\ \mathrm{Cr}(1)-\mathrm{O}(1) & 1.717(10) \\ \mathrm{N}(1)-\mathrm{C}(1) & 1.36(2) \\ \mathrm{N}(1)-\mathrm{C}(5) & 1.37(2) \\ \mathrm{O}(2)-\mathrm{Mn}(1) \# 1 & 2.108(11) \\ \mathrm{O}(1)-\mathrm{Mn}(1)-\mathrm{O}(2) \# 1 & 90.1(4) \\ \mathrm{O}(1)-\mathrm{Mn}(1)-\mathrm{N}(2) & 94.5(5) \\ \mathrm{O}(2) \# 1-\mathrm{Mn}(1)-\mathrm{N}(2) & 111.8(4) \\ \mathrm{O}(1)-\mathrm{Mn}(1)-\mathrm{N}(3) & 110.2(4) \\ \mathrm{O}(2) \# 1-\mathrm{Mn}(1)-\mathrm{N}(3) & 90.9(5) \\ \mathrm{N}(2)-\mathrm{Mn}(1)-\mathrm{N}(3) & 146.6(4) \\ \mathrm{O}(1)-\mathrm{Mn}(1)-\mathrm{N}(1) & 159.9(4) \\ \mathrm{O}(2) \# 1-\mathrm{Mn}(1)-\mathrm{N}(1) & 82.6(4) \\ \mathrm{N}(2)-\mathrm{Mn}(1)-\mathrm{N}(1) & 71.2(5) \\ \mathrm{N}(3)-\mathrm{Mn}(1)-\mathrm{N}(1) & 88.7(5) \\ \mathrm{O}(1)-\mathrm{Mn}(1)-\mathrm{N}(4) & 93.3(4) \\ \mathrm{O}(2) \# 1-\mathrm{Mn}(1)-\mathrm{N}(4) & 162.1(5) \\ \mathrm{N}(2)-\mathrm{Mn}(1)-\mathrm{N}(4) & 85.4(5) \\ \mathrm{N}(3)-\mathrm{Mn}(1)-\mathrm{N}(4) & 71.4(5) \\ \mathrm{N}(1)-\mathrm{Mn}(1)-\mathrm{N}(4) & 99.3(4) \\ \mathrm{O}(3)-\mathrm{Cr}(1)-\mathrm{O}(4) & 111.1(8) \\ \mathrm{O}(3)-\mathrm{Cr}(1)-\mathrm{O}(2) & 110.7(6) \\ \mathrm{O}(4)-\mathrm{Cr}(1)-\mathrm{O}(2) & 107.5(7) \\ \mathrm{O}(3)-\mathrm{Cr}(1)-\mathrm{O}(1) & 108.3(5) \\ \mathrm{O}(4)-\mathrm{Cr}(1)-\mathrm{O}(1) & 110.9(6) \\ \mathrm{O}(2)-\mathrm{Cr}(1)-\mathrm{O}(1) & 108.3(5) \\ \mathrm{C}(1)-\mathrm{N}(1)-\mathrm{C}(5) & 118.2(13) \\ \mathrm{C}(1)-\mathrm{N}(1)-\mathrm{Mn}(1) & 126.4(10) \\ \mathrm{C}(5)-\mathrm{N}(1)-\mathrm{Mn}(1) & 114.1(11) \\ \mathrm{C}(6)-\mathrm{N}(2)-\mathrm{C}(10) & 119.9(14) \\ \mathrm{C}(6)-\mathrm{N}(2)-\mathrm{Mn}(1) & 117.2(11) \\ \mathrm{C}(10)-\mathrm{N}(2)-\mathrm{Mn}(1) & 122.7(10) \\ \mathrm{C}(17)-\mathrm{N}(3)-\mathrm{C}(13) & 117.1(14) \\ \mathrm{C}(17)-\mathrm{N}(3)-\mathrm{Mn}(1) & 118.6(11) \\ \mathrm{C}(13)-\mathrm{N}(3)-\mathrm{Mn}(1) & \\ \mathrm{C}(22)-\mathrm{N}(4)-\mathrm{C}(18) & \mathrm{1}) \\ \mathrm{C}(22)-\mathrm{N}(4)-\mathrm{Mn}(1) & \mathrm{C}(18)-\mathrm{N}(4)-\mathrm{Mn}(1) \\ \mathrm{Cr}(1)-\mathrm{O}(1)-\mathrm{Mn}(1) & \mathrm{Cr}(1)-\mathrm{O}(2)-\mathrm{Mn}(1) \# 1 \\ & \end{array}$

\section{Conclusions}

The novel $\mathrm{Mn}$ complex $\mathrm{C}_{24} \mathrm{H}_{18} \mathrm{CrMnN}_{4} \mathrm{O}_{5}$ was investigated by hydrothermal and its crystal structure was characterized using X-ray diffraction technology.

\section{Acknowledgments}

Weifang University Doctor Foundation(No. 2019BS06). Jilin University National Key Laboratory Foundation (201323 and 2011-13). "Biochemistry and Molecular Biology" Shandong Province Key Laboratory (Weifang University).

\section{References}

1. Zhang X.M., Tong M.L., Chen X.M., (2002) Hydroxylation of N-Heterocycle Ligands Observed in Two Unusual Mixed-Valence CuI/CuII Complexes, Angew. Chem., Int. Ed., 41: 1029-1031.

2. Feng S.H., Xu R.R., (2001) New materials in hydrothermal synthesis, Chem. Res. J., 34: 239-247.

3. Qi Y.F., Wang X.L., Wang E.B., Qin C., Na H., (2005) Two new metal coordination polymers containing mixed ligands: hydrothermal synthesis and crystal structures of $\left[\mathrm{Co}_{2}(1,2,4,5\right.$-btc $\left.)(\text { phen })_{2}\left(\mathrm{H}_{2} \mathrm{O}\right)_{6}\right] \cdot 2 \mathrm{H}_{2} \mathrm{O}$ and $\left[\mathrm{Mn}_{3}(1,2,4-\mathrm{btc})_{2}(\text { phen })_{2}\left(\mathrm{H}_{2} \mathrm{O}\right)_{2}\right]_{\mathrm{n}}$, Journal of Coordination Chemistry, 58: 1289-1297.

4. Sundaramoorthy M., Kishi K., Gold M.H., Poulos T.L., (1994) The crystal structure of manganese peroxidase from Phanerochaete chrysosporium at 2.06-A resolution, J. Biol. Chem., 269: 32759-32767.

5. Borgstahl G.E., Parge H.E., Hickey M.J., Beyer Jr. W.F., Hallewell R.A., Tainer J.A., (1992) The 
structure of human mitochondrial manganese superoxide dismutase reveals a novel tetrameric interface of two 4-helix bundles, Cell, 71: 107-118.

6. Lee J.O., Bankston L.A., Arnaout M.A., Liddington R.C., (1995) Two conformations of the integrin A-domain: a pathway for activation Structure, 3: 1333-1340.

7. Liu, J.L., Chen,Y.C., Tong, M.L., (2018) Symmetry Strategies for High Performance Lanthanide-Based Single-Molecule Magnets, Chem. Soc. Rev., 47(7): 2431-2453.

8. BARBARA HACHULA, MONIKA PE DRAS, MARIA NOWAK, JOACHIM KUSZ, DANUTA SKRZYPEK, JERZY BOREK and DANUTA PENTAK. Synthesis, crystal structure, spectroscopic, and magnetic properties of a manganese(II) methoxyacetate complex $\left[\mathrm{Mn}\left(\mathrm{C}_{6} \mathrm{O}_{6} \mathrm{H}_{10}\right)\left(\mathrm{H}_{2} \mathrm{O}\right)\right]_{\mathrm{n}}$, (2010) Journal of Coordination Chemistry, 63: 67-78.

9. Yang H.L., Chen F., Zhang D., He X., Zhang X.Q., (2019) Syntheses, Crystal Structures, Thermal Stabilities and Fluorescence Properties of Silver(I) and Cadmium(II) Complexes Based on 1,2,4-Benzenetricarboxylic Acid and 2,2'-Bipyridine, Chinese Journal of Inorganic Chemistry, 35(9): 1579-1585.

10. Liu Zh.Q., Cao Sh.H., Zhang Z., Wu J.F., Zhao Y., Sun W.Y., (2019) Metal-Organic Frameworks with 2,6-Di(1H-imidazol-1-yl)naphthalene and Dicarboxylate Ligands: Synthesis, Crystal Structure and Photoluminescence Sensing Property, Chinese Journal of Inorganic Chemistry, 35(11): 2145-2151.

11. Zhang L., Jiang C., Guo L.F., Zhang X.L., Lin X.Q., Kang J., Sun W.M., (2021) Synthesis, Characterization, Antitumor Activity, and Theoretical Calculations of Co Complex Based on Pyridine-2,6-dicarboxylic Acid, Chinese Journal of Inorganic Chemistry, 37(2): 368-374. 